\title{
ATF3 suppresses ESCC via downregulation of ID1
}

\author{
JIAN LI ${ }^{1}$, ZISHAN YANG ${ }^{2}$, ZHIUGUO CHEN ${ }^{2,3}$, YONGHUA BAO ${ }^{2,4}$, HUIJUAN ZHANG $^{5}$, \\ XINHUI FANG ${ }^{1}$ and WANCAI YANG ${ }^{2,6,7}$
}

\begin{abstract}
${ }^{1}$ Department of Gastroenterology, People's Hospital of Zhengzhou University, Henan Provincial People's Hospital, Zhengzhou, Henan 450052; ${ }^{2}$ Laboratory for Cancer Signal Transduction, Xinxiang Medical University, Xinxiang, Henan 453003; ${ }^{3}$ Department of Histology, School of Medicine, Wuhan University, Wuhan, Hubei 430072; ${ }^{4}$ Department of Immunology, Xinxiang Medical University, Xinxiang, Henan 453003; ${ }^{5}$ Department of Gastroenterology, Seventh People's Hospital of Zhengzhou City, Zhengzhou, Henan 45000; ${ }^{6}$ Department of Pathology, Xinxiang Medical University, Xinxiang, Henan 453003, P.R. China; ${ }^{7}$ Department of Pathology, College of Medicine, University of Illinois at Chicago, Chicago, IL 60612, USA
\end{abstract}

Received May 11, 2015; Accepted June 7, 2016

DOI: 10.3892/ol.2016.4832

\begin{abstract}
Esophageal cancer is one of the most prevalent forms of cancer and has a particularly high mortality rate due to early metastasis; however, the underlying mechanisms of its formation and progression remain unclear. The present study performed immunohistochemical analysis and observed that the expression of activating transcription factor 3 (ATF3) was reduced in esophageal squamous cell carcinoma (ESCC) in comparison with non-tumor adjacent tissues. By contrast, inhibitor of DNA binding 1 (ID1) was overexpressed in ESCC tissues, demonstrating an inverse correlation with ATF3 (P<0.01). In ESCC EC109 and KYSE450 cells lines, transfection with an ATF3-overexpression plasmid resulted in the inhibition of cell proliferation, motility and migration, which was associated with the induction of E-cadherin expression and inhibition of cyclin D1 and Twist. Notably, ATF3 exerted an inverse regulatory interaction with ID1. The results of the present study provide additional evidence of the tumor suppressive features of ATF3 and demonstrate a novel mechanism of ATF3-mediated inhibition of cancer metastasis in esophageal cancer.
\end{abstract}

Correspondence to: Professor Wancai Yang, Department of Pathology, Xinxiang Medical University, 601 East Jinsui Avenue, Xinxiang, Henan 453003, P.R. China

E-mail:wyang06@uic.edu

Abbreviations: ESCC, esophageal squamous cell carcinoma; ATF3, activating transcription factor 3; ID1, inhibitor of DNA binding 1; STAT3, signaling transducer and activator of transcription 3

Key words: esophageal squamous cell carcinoma, activating transcription factor 3, inhibitor of DNA binding 1, proliferation, metastasis

\section{Introduction}

Activating transcription factor 3 (ATF3) is a member of the ATF/cyclic AMP-responsive element binding protein (CREB) family of transcription factors and has been demonstrated to form dimers with other ATF/CREB proteins, including c-Jun, ATF2, Jun D and Jun B (1). As a transcription factor, ATF3 is critical in cell growth, apoptosis and carcinogenesis (1). Notably, ATF3 has been reported to serve controversial roles in oncogenesis and tumor suppression depending on the context and cell type. Several studies support the oncogenic role of ATF3. For example, ATF3 has been reported to be overexpressed in breast cancer (2), prostate cancer (3) and Hodgkin lymphomas (4), and high expression is an indicator of poor prognosis of patients with prostate cancer (3). In vivo and in vitro studies have also demonstrated that overexpression of ATF3 promotes cancer cell proliferation and metastasis in prostate cancer $(5,6)$, and is associated with upregulation of Slug, fibronectin-1 and TWIST1 transcripts, which are important regulators of cell-extracellular matrix or cell-cell interactions $(5,6)$. In addition, ATF3 overexpression results in the binding of ATF3 to the GADD153 promoter, which subsequently represses its transcription in cervical cancer HeLa cells, providing a potential pathway through which ATF3 is able to promote cancer cell survival (7). In contrast to the aforementioned studies, growing evidence suggests that ATF3 is able to suppress tumorigenesis. For example, it has been demonstrated that ATF3 expression levels are reduced in human colorectal cancer, and overexpression of ATF3 exhibits tumor suppressive roles, such that the protein reduces metastatic potential and promotes apoptosis in various cell lines to inhibit carcinogenesis $(8,9)$. In addition, ATF3 is able to suppress the oncogenic function of mutant p53 in lung cancer (10). The possible role of ATF3 as a tumor suppressor is supported by its established role in transforming growth factor- $\beta$ (TGF- $\beta$ ) signaling (11). TGF- $\beta$ is a potent tumor suppressor in epithelial cells that signals via Smad3 activation to directly induce ATF3 (11). Smad3 and ATF3 subsequently form a complex, which binds to the promoter of inhibitor of DNA binding 1 
(ID1) and directly mediates its repression (11). Furthermore, ATF3 may be activated by a range of anticancer compounds, including non-steroidal anti-inflammatory drugs, curcumin, dietary compounds resveratrol and genistein, progesterone and the phosphatidylinositol inhibitor LY294002 (1,12). Inversely, resveratrol and genistein also suppress ID1 expression (12).

ID1 is a member of the ID protein superfamily, which belongs to the helix-loop-helix transcription factor family $(13,14)$. ID1 is ubiquitously expressed in a number of tissues and functions in a wide range of cellular processes, including proliferation, cell differentiation, senescence and apoptosis (15). Growing evidence suggests that ID1 is an oncogene and is critical in promoting tumor invasion and development, as it is overexpressed in human cancer of the pancreas, thyroid, breast, cervix, ovary, prostate, esophagus and lung, and high expression of ID1 is associated with a poor prognosis (16-18). Furthermore, ID1 is able to promote cell survival and induce cancer cell growth, which may be associated with ATF3 (19).

Recent studies have reported that ATF3 was downregulated in esophageal squamous cell carcinoma (ESCC) compared with paired non-cancerous tissues, and that lower ATF3 expression in tumors was significantly correlated with shorter survival time $(20,21)$. Furthermore, increased expression of ATF3 inhibited ESCC cell growth and invasion in vitro and in nude mice via p53 signaling (21). However, it is unclear whether ESCC tumor inhibition by ATF3 occurs through ID1 repression. ESCC is one of the most common malignancies in worldwide. There were about 477,900 new cases and 375,000 death of ESCC in China (22). The present study aimed to determine the association between ATF3 and ID1 in ESCC tissues and in vitro by manipulating ATF3 expression.

\section{Materials and methods}

Human samples and immunohistochemical staining. A total of 36 pairs of ESCC tissues and their adjacent non-cancer tissues were obtained from the Tissue Bank of the Laboratory for Cancer Signal Transduction, Xinxiang Medical University (Xinxiang, China). All procedures were approved by the Institutional Review Board of Xinxiang Medical University.

Immunohistochemical staining was conducted as previously described (23). In brief, formalin-fixed, paraffin-embedded tissues were sectioned and deparaffinized with xylene, rehydrated with gradient ethanol and distilled water, and were subsequently blocked with serum and incubated with anti-AFT3 (1:100, ca no. ab191513) and anti-ID1 (1:100, cat no. ab134163) primary antibodies (Abcam, Cambridge, MA, USA) at $4^{\circ} \mathrm{C}$ overnight. The sections were then incubated with a biotinylated goat anti-rabbit IgG antibody, dilution (1:200; cat no. PI-1000; Vector Laboratories, Inc., Burlingame, CA, USA), and a VECTASTAIN Elite ABC kit (Vector Laboratories, Inc., Burlingame, CA, USA) was used according to the manufacturer's protocol. The sections were finally stained with 3,3'-diaminobenzidine. Immunohistochemical staining was evaluated based on immunostaining intensities (absent or weak, moderate and strong) as previously described (23).

Cell culture and transfection. Human ESCC EC109 and KYSE450 cell lines obtained from American Type Culture
Collection (Manassas, VA, USA) were cultured in Dulbecco's modified Eagle's medium containing $10 \%$ fetal calf serum (Invitrogen; Thermo Fisher Scientific, Inc., Waltham, MA, USA). All cells were incubated at $37^{\circ} \mathrm{C}$ in a humidified atmosphere containing 5\% $\mathrm{CO}_{2}$. An overexpression plasmid for ATF3 was constructed as follows: Human ATF3 cDNA was amplified and inserted into a pcDNA3-Flag vector. The expression plasmid (pFlag-ATF3) was transfected into the ESCC cell lines using Lipofectamine ${ }^{\circledR} 2000$ (Invitrogen; Thermo Fisher Scientific, Inc.) following the manufacturer's protocol. An empty vector (pFlag-cDNA3) was also transfected into the ESCC cells, which served as a negative control.

Cell proliferation assay. 3-(4,5-dimethylthiazol-2-yl) 5-(3-carb oxymethoxyphenyl)-2-(4-sulfophenyl)-2H-tetrazolium (MTS) assay was performed to determine cytotoxicity of the EC109 and KYSE450 cells following transfection with pFlag-ATF3 using Lipofectamine 2000. pFlag-cDNA3 was used as a control for comparison. Following 24, 36 and $48 \mathrm{~h}$, cell proliferation was determined by MTS assay using the CellTiter $96^{\circledR}$ Non-Radioactive Cell Proliferation Assay kit (Promega Corporation, Madison, WI, USA) according to the manufacturer's protocol. The remaining viable cells with MTS uptake were determined by measuring the optical density at $570 \mathrm{~nm}$ using an enzyme-linked immunosorbent assay reader (Molecular Devices, LLC, Sunnyvale, CA, USA). Values are presented as the mean \pm standard deviation. At least three measurements were read, and the experiments were conducted three times independently.

Wound healing and transwell assays. As previously described (24), EC109 and KYSE450 cells transiently transfected with pFlag-ATF3 plasmid or the pFlag-cDNA3 empty vector were seeded in a $100-\mathrm{mm}$ Petri dish. A wound was made by scratching on the bottom of the dish, followed by a $36 \mathrm{~h}$ incubation at $37^{\circ} \mathrm{C}$. The wound healing status was checked under an inverse microscope. Cell migration was detected using a Transwell plate (Corning Life Sciences, Lowell, MA, USA). Approximately $1 \times 10^{3}$ EC109 cells were transiently transfected with the pFlag-ATF3 plasmid or the pFlag-cDNA3 empty vector, and were subsequently seeded into the Transwell plate. The cells were cultured in Dulbecco's modified Eagle's medium (Invitrogen; Thermo Fisher Scientific, Inc., Waltham, MA, USA) containing $10 \%$ fetal calf serum (Invitrogen, Carlsbad, CA). The migrated cells were stained with 4',6-diamidino-2-phenylindole and counted under an inverse fluorescence microscope at $48 \mathrm{~h}$ post-seeding.

Immunoblotting. For immunoblotting, human ESCC EC109 and KYSE459 cells were collected $48 \mathrm{~h}$ post-transfection with pFlag-ATF3 or pFlag-cDNA3 empty vector. Cells were lysed using $1 \mathrm{X}$ radioimmunoprecipitation assay (RIPA) buffer (Upstate Biotechnology, Inc., Lake Placid, NY, USA) containing a protease inhibitor cocktail (Sigma-Aldrich, St. Louis, MO, USA). Following cell lysis, $45 \mu \mathrm{g}$ protein was loaded on a $10 \%$ sodium dodecyl sulfate (SDS) gel followed by transfer to a polyvinylidene fluoride membrane. The membrane was probed with antibodies against Flag (cat no. ab49763, dilution 1:1000), ATF3 (cat no. ab191513, dilution 1:250), ID1 (cat no. ab134163, 1:250), cyclin D1 (cat no. ab134175, 
Table I. Expression status of ATF3 and ID1 and their correlation in esophageal squamous cell carcinoma tissues.

\begin{tabular}{lcccc}
\hline Protein & $\begin{array}{c}\text { Absent/weak, } \\
\mathrm{n}(\%)\end{array}$ & $\begin{array}{c}\text { Moderate, } \\
\mathrm{n}(\%)\end{array}$ & $\begin{array}{c}\text { Strong, } \\
\mathrm{n}(\%)\end{array}$ & $\begin{array}{c}\text { Total, } \\
\mathrm{n}(\%)\end{array}$ \\
\hline ATF3 & $10(27.7)$ & $17(47.2)$ & $9(25.0)$ & $36(100.0)$ \\
ID1 & $4(11.1)$ & $10(27.8)$ & $22(61.1)$ & $36(100.0)$
\end{tabular}

$\mathrm{X}^{2}=9.84 ; \mathrm{P}<0.01$ in the correlation analysis of ATF3 expression and ID1 expression in ESCC. $\mathrm{n}$ refers to the number of cases studied in each group. ATF3, activating transcription factor 3; ID1, inhibitor of DNA binding 1.

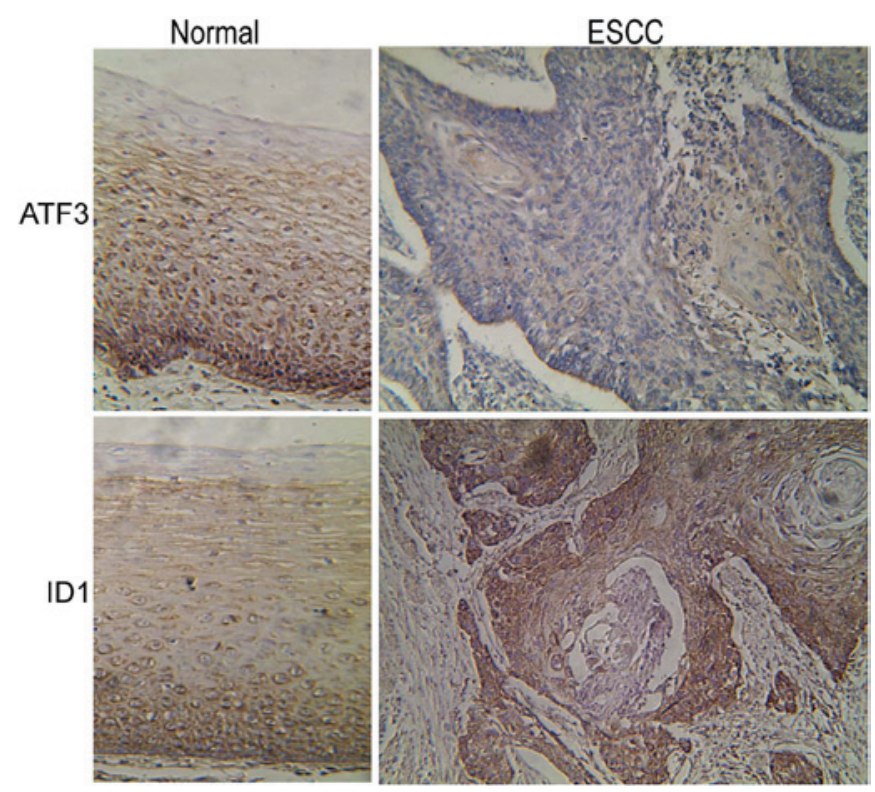

Figure 1. Immunohistochemical analysis of ATF3 and ID1 expression in normal esophageal epithelial and ESCC. ATF3 expression was reduced in ESCC tissues, whilst ID1 expression was increased in ESCC tissues compared with the normal esophageal epithelial cells. Magnification, x200. ATF3, activating transcription factor 3; ID1, inhibitor of DNA binding 1; ESCC, esophageal squamous cell carcinoma.

dilution 1:250), E-cadherin (cat no. ab40772, dilution 1:300; Abcam), phosphorylated (p)-STAT3 (cat no. 9131s, dilution 1:300), Twist (cat no. 4119s, 1:300; Cell Signaling Technology, Inc., Danvers, MA, USA), glyceraldehyde 3-phosphate dehydrogenase (cat no. G8795, dilution 1:500) and $\beta$-actin (cat no. A8481, dilution 1:1000; Sigma-Aldrich). Secondary antibodies (cat no. SC-2004, goat anti-rabbit IgG-HRP, dilution 1:1000; cat no. SC-2005, goat anti-mouse IgG-HRP, dilution 1:1000) were purchased from Santa Cruz Biotechnolog, Dallas, TX, USA. The signals were visualized using an enhanced chemiluminescence kit (Beyotime Institute of Biotechnology, Haimen, China) according to the manufacturer's protocol.

Quantitative reverse transcriptional PCR (RT- $q P C R)$ assay. To determine the alterations of ID1 at mRNA levels, the EC109 and KYSE450 cells transfected with pFlag-ATF3 or pFlag-cDNA3 were collected after $48 \mathrm{~h}$ post transfection. Total RNA were extracted and RT-qPCR were conducted to determine the changes of ID1 mRNA using the following
A

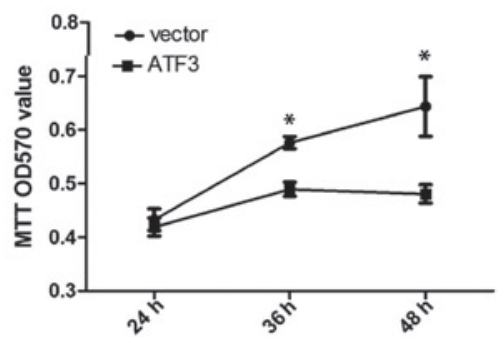

B

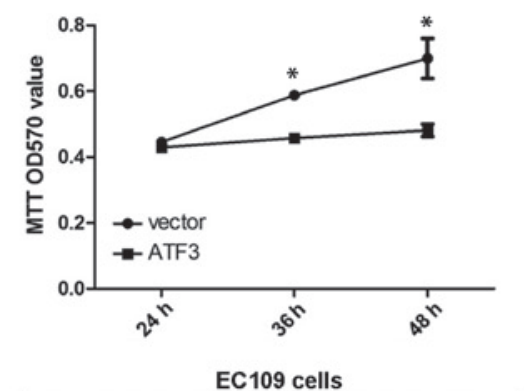

C

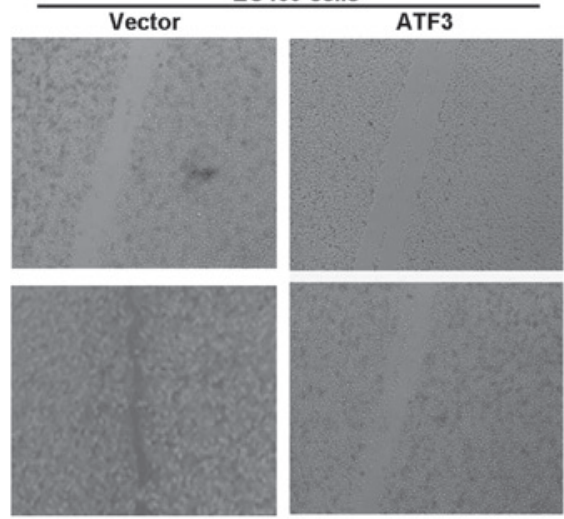

KYSE450 cells

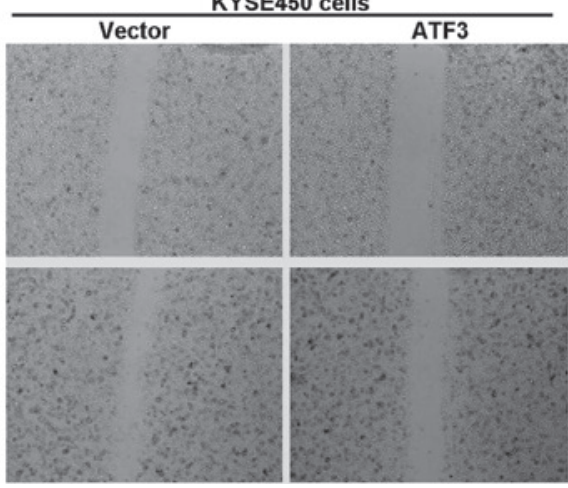

Figure 2. Increased expression of ATF3 inhibits ESCC cell proliferation and motility. Overexpression of ATF3 by transfection with ATF3 expression plasmid inhibited (A) EC109 and (B) KYSE450 cell proliferation after 36 and $48 \mathrm{~h}$ compared with the cells transfected with the empty vector ( $\mathrm{P}<0.05$ vs. empty vector). (C) Wound healing assay determined that the overexpression of ATF3 inhibited cell motility. ATF3, activating transcription factor 3; OD, optical density.

primers: ID1-forward: TGGATGGCGGGTTTCAGATG, ID1-reverse: TCTTCGGTCAGACGATTGACA. The detailed procedure was reported previously (23).

Co-immunoprecipitation assay. EC109 cells were transfected with pFlag-ATF3 or pFlag-cDNA3 for $48 \mathrm{~h}$. The cells were collected and incubated on ice for 15 min in RIPA lysis buffer supplemented with protease inhibitor cocktail. Total cell lysate was centrifuged at $8,000 \mathrm{xg}$ for $15 \mathrm{~min}$ at $4^{\circ} \mathrm{C}$. A total of $300 \mathrm{mg}$ 
A
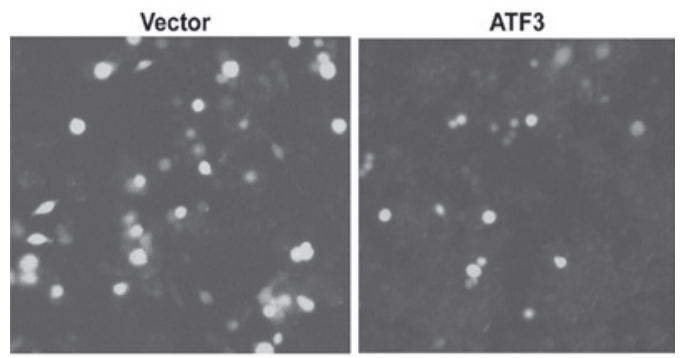

B

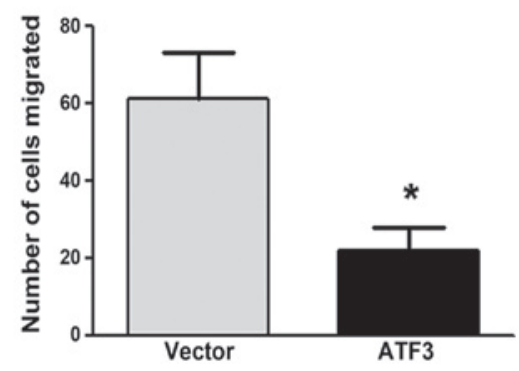

Figure 3. Increased expression of ATF3 inhibits ESCC cell migration. (A) Transwell assay determined that transfection with the ATF3-overexpression plasmid inhibited EC109 cell migration after $48 \mathrm{~h}$ compared with the cells transfected with the empty vector. (B) Quantification of the migrated cells between the cells transfected with the empty vector or ATF3 plasmids ( ${ }^{*} \mathrm{P}<0.05$ vs. empty vector group). ATF3, activating transcription factor 3 ; ESCC, esophageal squamous cell carcinoma.

supernatant was incubated with anti-Flag antibody (GenScript Corporation, Scotch Plains, NJ, USA) overnight at $4^{\circ} \mathrm{C}$ on a rotator, followed by the addition of Protein A/G PLUS-Agarose (Santa Cruz Biotechnology, Inc.) for $2 \mathrm{~h}$ at $4^{\circ} \mathrm{C}$. The immunocomplex was separated by $12 \%$ SDS-polyacrylamide gel electrophoresis. The PVDF membrane was probed with anti-ID1 antibody to detect the ATF3/ID1 complex.

Statistical analysis. Data were presented as mean \pm standard deviation (SD), the Student-t test was used for groups' quantity comparison, and Chi-square test was used for correlation analysis. $\mathrm{P}<0.05$ was considered to indicate a statistically significant difference.

\section{Results}

Inverse correlation between ATF3 and ID1 expression in the ESCC tissues. Using immunohistochemical staining, it was observed that ATF3 was expressed in the ESCC and adjacent non-tumor tissues; however, ATF3 expression was reduced in the ESCC tissues compared with the adjacent non-tumor tissues (Fig. 1). By contrast, ID1 was overexpressed in ESCC tissues compared with the adjacent non-tumor tissues (Fig. 1). These results therefore demonstrate a significant inverse correlation between ATF3 and ID1 expression in the ESCC tissues (Table I; $X^{2}=9.84 ; \mathrm{P}<0.01$ ).

ATF3 inhibits cell proliferation, motility and migration in ESCC cells. To determine the roles of ATF3 in ESCC cells, the ATF3 overexpression plasmid was transfected in to the EC109 and KYSE450 cells. It was observed that increased ATF3 expression significantly inhibited cell proliferation in each ESCC cell line (Fig. 2A and B). Wound healing assay demonstrated that ATF3 overexpression inhibited ESCC
A
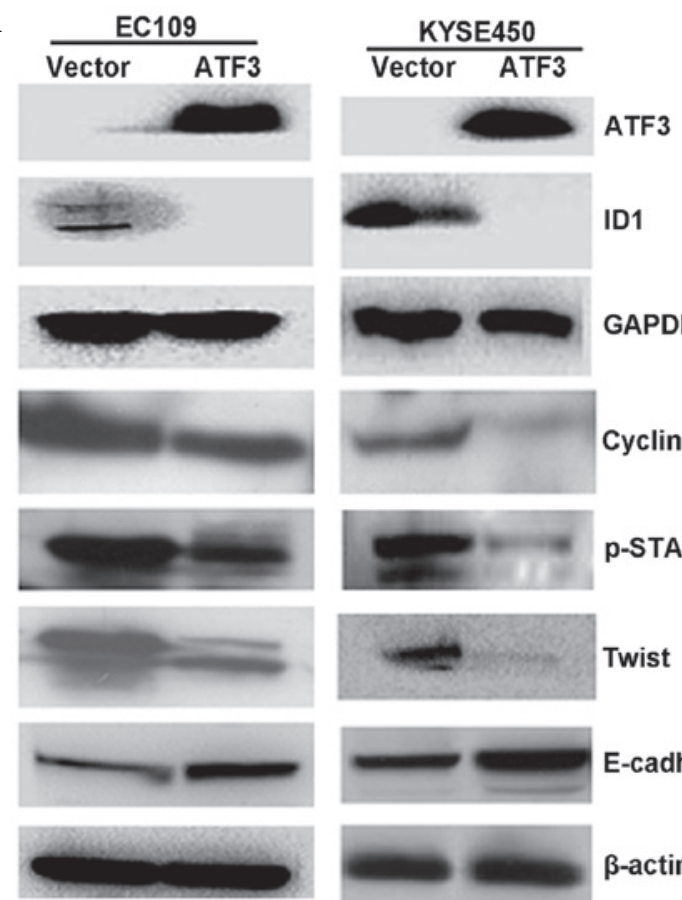

TF3

1

GAPDH
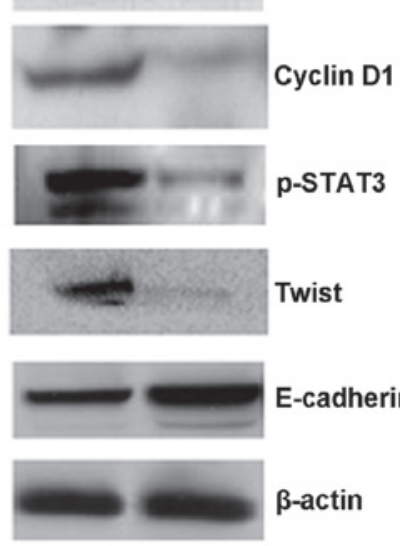

B

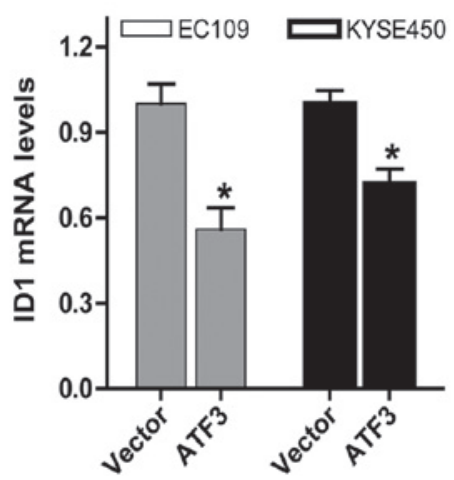

Figure 4. (A) Increased expression of ATF3 affected the expression of proteins associated with cell proliferation and epithelial-mesenchymal transition/metastasis and (B) repressed ID1 mRNA levels in esophageal squamous cell carcinoma cells ( $\mathrm{P}<0.05$ vs. empty vector groups). ATF3, activating transcription factor 3; ID1, inhibitor of DNA binding 1; GAPDH, glyceraldehyde 3-phosphate dehydrogenase; p-STAT3, phosphorylated signal transducer and activator of transcription 3 .

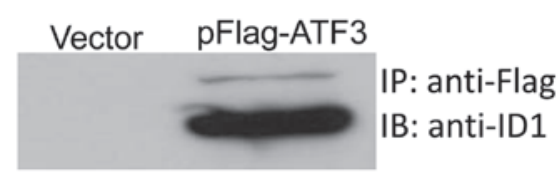

Figure 5. Co-immunoprecipitation assay demonstrated that ATF3 physically binds with ID1. ESCC cell line EC109 cells were transfected with the pFlag-ATF3 plasmid and harvested $48 \mathrm{~h}$ post-transfection. The cell lysate was pulled down by anti-flag antibodies and the immunocomplex was probed by anti-ID1 antibodies.

cell motility (Fig. 2C), and Transwell assay determined that ATF3 significantly inhibited ESCC cell migration $(\mathrm{P}<0.05)$ (Fig. 3).

To determine the mechanisms underlying these results, immunoblotting was performed. The results demonstrated that ATF3 repressed ID1 expression. In addition, ATF3 
downregulated the expression of cyclin D1, p-STAT3 and TWIST, and upregulated the expression of E-cadherin (Fig. 4A).

ATF3 represses IDI expression in ESCC cells. The present study subsequently investigated the effects of ATF3 on ID1 expression by transfecting ATF3 overexpression plasmids into ESCC EC109 and KYSE450 cells. The ID1 mRNA levels and their interactions were subsequently determined, and the results demonstrated that increased expression of ATF3 significantly suppressed ID1 expression at the mRNA level (Fig. 4B) as determined by reverse transcription-quantitative polymerase chain reaction, which was consistent with the inhibition of ID1 at the protein level (Fig. 4A).

To further investigate the interaction between ATF3 and ID1, co-immunoprecipitation assay was performed. Anti-Flag antibodies were used to pull down the immune-complex and anti-ID1 antibodies were used to probe the complex. As presented in Fig. 5, there was a strong band in the precipitated immune-complex, therefore suggesting that the ATF3 and ID1 proteins had bound together.

\section{Discussion}

Esophageal cancer is one of the most prevalent forms of cancer worldwide and is frequently fatal, with a 5-year survival rate of $<20 \%$ (25). The underlying mechanisms of carcinogenesis and progression remain largely unknown. Recent studies using genome-wide analysis and whole genome sequencing have identified several signal nucleotide polymorphisms (SNPs) or mutations associated with ESCC (26-29); however, the majority of these do not have biological functions. Thus, increasing attention is being focused on the identification of oncogenes or tumor suppressors. The present study investigated the transcription factor ATF3 and observed that its expression was reduced in ESCC, and that increased expression of ATF3 resulted in tumor inhibition, characterized by suppression of cell proliferation, motility and migration. In addition, it was noted that ATF3 also negatively regulated the oncogene ID1, and repressed the expression of cyclin D1, STAT3 and TWIST.

Controversial roles of ATF3 have been reported in various types of cancer in which ATF3 exhibits oncogenic or suppressive functions in cancer formation and progression. Consistent with a recent study of ESCC (21), the results of the current study demonstrated that ATF3 expression was lower in the ESCC tissues compared with the adjacent non-tumor tissues, and the expression levels negatively correlated with cancer differentiation, thus indicating the tumor suppressive functions of ATF3 in ESCC. By contrast, expression levels of the oncogene ID1 were higher in the ESCC tissues compared with the adjacent non-tumor tissues. ATF3 and ID1 expression demonstrated a significant inverse correlation in the ESCC tissues. In vitro experiments further indicated that increased expression of ATF3 repressed ID1 expression at the protein and mRNA level in ESCC cells, and that there was a negative regulatory interaction between ATF3 and ID1, supported by results from co-immunoprecipitation assays. The present study therefore provides additional evidence that ATF3 inhibits ID1 expression through a protein-protein interaction.
Tumor formation is understood to result from uncontrollable cell growth, which is associated with overexpression of cell cycle regulators (30), including cyclin dependent kinases, cyclins (e.g. cyclin D1) and cyclin dependent kinase inhibitors. Numerous studies have reported that cyclin D1 is overexpressed in cancer tissues and inhibition of cyclin D1 expression in vitro leads to cell proliferation inhibition $(31,32)$. In the present study, it was demonstrated that increased expression of ATF3 repressed cyclin D1 expression in the ESCC cells. Furthermore, STAT3 is known to serve a crucial role in carcinogenesis, particularly the activated form of STAT3, p-STAT3 $(33,34)$. The current study observed that increased ATF3 expression was able to suppress the levels of p-STAT3 in the ESCC cell lines, and the downregulation of cyclin D1 and p-STAT3 partially led to inhibition of ESCC cell proliferation in vitro.

Cancer-associated mortality is primarily caused by metastasis, and the latter is linked to the upregulation of TWIST and downregulation of E-cadherin (35-40). A number of studies have reported that loss of E-cadherin expression increases cancer metastasis by separating cancer cells from another, thus activating specific downstream signal transduction pathways resulting in epithelial-mesenchymal transition (41-45). Perturbation of E-cadherin-mediated cell adhesion is implicated in the progression of tumors, poor prognosis and metastasis (35-40). An increasing body of evidence indicates the importance of E-cadherin in cancer metastasis and development, and the possible regulation of E-cadherin by glycogen synthase kinase 3 $\beta$, TWIST/Snail and Akt/protein kinase B (41-43). Maintaining the expression of E-cadherin may prevent tumor invasion and metastasis and restore epithelial morphology $(44,45)$. In the present study, it was observed that increasing ATF3 expression upregulated E-cadherin expression in the ESCC cells and downregulated the expression of Twist, resulting in the inhibition of ESCC cell migration in vitro.

In conclusion, the current study demonstrated that ATF3 expression was decreased and ID1 expression was overexpressed in ESCC tissues; however, increasing ATF3 expression led to inhibition of cell proliferation, migration and motility in vitro, which was associated with the upregulation of E-cadherin and the downregulation of cyclin D1 and Twist, and most importantly highlighted the inverse regulatory interaction between ATF3 and ID1. These results provide additional evidence of the tumor suppressive features of ATF3 and demonstrate the novel mechanism of ATF3-mediated inhibition of cancer metastasis in esophageal cancer.

\section{Acknowledgements}

The current study was supported in part by the Provincial Natural Science Foundation (grant no. 122300410401) from the Science and Technology Department of Henan Province, China, and the US Chinese Anti-Cancer Association, CA, USA (grant no. USCACA-TIGM-1).

\section{References}

1. Thompson MR, Xu D and Williams BR: ATF3 transcription factor and its emerging roles in immunity and cancer. J Mol Med (Berl) 87: 1053-1060, 2009. 
2. Yin X, Dewille JW and Hai T: A potential dichotomous role of ATF3, an adaptive-response gene, in cancer development Oncogene 27: 2118-2127, 2008.

3. Pelzer AE, Bektic J, Haag P, Berger AP, Pycha A, Schäfer G, Rogatsch $\mathrm{H}$, Horninger $\mathrm{W}$, Bartsch $\mathrm{G}$ and Klocker $\mathrm{H}$ : The expression of transcription factor activating transcription factor 3 in the human prostate and its regulation by androgen in prostate cancer. J Urol 175: 1517-1522, 2006.

4. Janz M, Hummel M, Truss M, Wollert-Wulf B, Mathas S, Jöhrens K, Hagemeier C, Bommert K, Stein H, Dörken B and Bargou RC: Classical Hodgkin lymphoma is characterized by high constitutive expression of activating transcription factor 3 (ATF3), which promotes viability of Hodgkin/Reed-Sternberg cells. Blood 107: 2536-2539, 2006.

5. Ling MT, Lau TC, Zhou C, Chua CW, Kwok WK, Wang Q, Wang $\mathrm{X}$ and Wong YC: Overexpression of Id-1 in prostate cancer cells promotes angiogenesis through the activation of vascular endothelial growth factor (VEGF). Carcinogenesis 26 : 1668-1676, 2005

6. Bandyopadhyay S, Wang Y, Zhan R, Pai SK, Watabe M, Iiizumi M, Furuta E, Mohinta S, Liu W, Hirota S, et al: The tumor metastasis suppressor gene Drg-1 down-regulates the expression of activating transcription factor 3 in prostate cancer. Cancer Res 66: 11983-11990, 2006.

7. Maytin EV, Ubeda M, Lin JC and Habener JF: Stress-inducible transcription factor $\mathrm{CHOP} / g a d d 153$ induces apoptosis in mammalian cells via p38 kinase-dependent and -independent mechanisms. Exp Cell Res 267: 193-204, 2001.

8. Yuan X, Yu L, Li J, Xie G, Rong T, Zhang L, Chen J, Meng Q, Irving AT, Wang D, et al: ATF3 suppresses metastasis of bladder cancer by regulating gelsolin-mediated remodeling of the actin cytoskeleton. Cancer Res 73: 3625-3637, 2013.

9. Jan YH, Tsai HY, Yang CJ, Huang MS, Yang YF, Lai TC, Lee CH, Jeng YM, Huang CY, Su JL, et al: Adenylate kinase- 4 is a marker of poor clinical outcomes that promotes metastasis of lung cancer by downregulating the transcription factor ATF3. Cancer Res 72: 5119-5129, 2012.

10. Wei S, Wang H, Lu C, Malmut S, Zhang J, Ren S, Yu G, Wang W, Tang DD and Yan C: The activating transcription factor 3 protein suppresses the oncogenic function of mutant p53 proteins. J Biol Chem 289: 8947-8959, 2014.

11. Kang Y, Chen CR and Massague J: A self-enabling TGFbeta response coupled to stress signaling: Smad engages stress response factor ATF3 for Id1 repression in epithelial cells. Mol Cell 11: 915-926, 2003.

12. Bottone FG Jr and Alston-Mills B: The dietary compounds resveratrol and genistein induce activating transcription factor 3 while suppressing inhibitor of DNA binding/differentiation-1. J Med Food 14: 584-593, 2011.

13. Perk J, Iavarone A and Benezra R: Id family of helixloop-helix proteins in cancer. Nat Rev Cancer 5: 603-614, 2005.

14. Benezra R, Davis RL, Lockshon D, Turner DL and Weintraub H: The protein Id: A negative regulator of helix-loop-helix DNA binding proteins. Cell 61: 49-59, 1990

15. Patel D, Morton DJ, Carey J, Havrda MC and Chaudhary J: Inhibitor of differentiation 4 (ID4): From development to cancer. Biochim Biophys Acta 1855: 92-103, 2014.

16. Castanon E, Bosch-Barrera J, López I, Collado V, Moreno M, López-Picazo JM, Arbea L, Lozano MD, Calvo A and Gil-Bazo I: Id1 and Id3 co-expression correlates with clinical outcome in stage III-N2 non-small cell lung cancer patients treated with definitive chemoradiotherapy. J Transl Med 11: 13, 2013.

17. Tang R, Hirsch P, Fava F, Lapusan S, Marzac C, Teyssandier I, Pardo J, Marie JP and Legrand O: High Id1 expression is associated with poor prognosis in 237 patients with acute myeloid leukemia. Blood 114: 2993-3000, 2009.

18. Schoppmann SF, Schindl M, Bayer G, Aumayr K, Dienes J, Horvat R, Rudas M, Gnant M, Jakesz R and Birner P: Overexpression of Id-1 is associated with poor clinical outcome in node negative breast cancer. Int J Cancer 104: 677-682, 2003.

19. Kashiwakura Y, Ochiai K, Watanabe M, Abarzua F, Sakaguchi M, Takaoka M, Tanimoto R, Nasu Y, Huh NH and Kumon H: Down-regulation of inhibition of differentiation-1 via activation of activating transcription factor 3 and Smad regulates REIC/Dickkopf-3-induced apoptosis. Cancer Res 68: 8333-8341, 2008.
20. Tan H, Zhang H, Xie J, Chen B, Wen C, Guo X, Zhao Q, Wu Z, Shen J, Wu J, et al: A novel staging model to classify oesophageal squamous cell carcinoma patients in China. Br J Cancer 110: 2109-2115, 2014.

21. Xie JJ, Xie YM, Chen B, Pan F, Guo JC, Zhao Q, Shen JH, Wu ZY, Wu JY, Xu LY and Li EM: ATF3 functions as a novel tumor suppressor with prognostic significance in esophageal squamous cell carcinoma. Oncotarget 5: 8569-8582, 2014.

22. Chen W, Zheng R, Baade PD, Zhang S, Zeng H, Bray F, Jemal A, Yu XQ and He J: Cancer statistics in China, 2015. CA Cancer J Clin 66: 115-32, 2016.

23. Li T, Yang W, Li M, Byun DS, Tong C, Nasser S, Zhuang M, Arango D, Mariadason JM and Augenlicht LH: Expression of selenium-binding protein 1 characterizes intestinal cell maturation and predicts survival for patients with colorectal cancer. Mol Nutr Food Res 52: 1289-1299, 2008.

24. Bi X, Pohl NM, Qian Z, Yang GR, Gou Y, Guzman G, Kajdacsy-Balla A, et al: Decorin-mediated inhibition of colorectal cancer growth and migration is associated with E-cadherin in vitro and in mice. Carcinogenesis 33: 326-330, 2012.

25. Parkin DM, Bray F, Ferlay J and Pisani P: Global cancer statistics, 2002. CA Cancer J Clin 55: 74-108, 2005.

26. Wang LD, Zhou FY, Li XM, Sun LD, Song X, Jin Y, Li JM, Kong GQ, Qi H, Cui J, et al: Genome-wide association study of esophageal squamous cell carcinoma in Chinese subjects identifies susceptibility loci at PLCE1 and C20orf54. Nat Genet 42: 759-763, 2010

27. Abnet CC, Freedman ND, Hu N, Wang Z, Yu K, Shu XO, Yuan JM, Zheng W, Dawsey SM, Dong LM, et al: A shared susceptibility locus in PLCE1 at 10q23 for gastric adenocarcinoma and esophageal squamous cell carcinoma. Nat Genet 42: 764-767, 2010.

28. Wu C, Hu Z, He Z, Jia W, Wang F, Zhou Y, Liu Z, Zhan Q, Liu Y, Yu D, et al: Genome-wide association study identifies three new susceptibility loci for esophageal squamous-cell carcinoma in Chinese populations. Nat Genet 43: 679-684, 2011.

29. Wu C, Wang Z, Song X, Feng XS, Abnet CC, He J, Hu N, Zuo XB, Tan W, Zhan Q, et al: Joint analysis of three genome-wide association studies of esophageal squamous cell carcinoma in Chinese populations. Nat Genet 46: 1001-1006, 2014.

30. Shtutman M, Zhurinsky J, Simcha I, Albanese C, et al: The cyclin D1 gene is a target of the beta-catenin/LEF-1 pathway. PNAS U.S.A. 96, 5522-5527, 1999

31. Wu S, Bao Y, Ma D, Zi Y, et al: Sodium selenite inhibits leukemia HL-60 cell proliferation and induces cell apoptosis by enhancing the phosphorylation of JNK1 and increasing the expression of p21 and p27. Int J Mol Med, 34, 1175-1179, 2014

32. Okumura H, Uchikado Y, Setoyama T, Matsumoto M, Owaki T, Ishigami $\mathrm{S}$ and Natsugoe $\mathrm{S}$ : Biomarkers for predicting the response of esophageal squamous cell carcinoma to neoadjuvant chemoradiation therapy. Surg Today 44: 421-428, 2014.

33. Kamran MZ, Patil P and Gude RP: Role of STAT3 in cancer metastasis and translational advances. Biomed Res Int 2013: 421821, 2013

34. Quante M, Varga J, Wang TC and Greten FR: The gastrointestinal tumor microenvironment. Gastroenterology 145: 63-78, 2013.

35. Doki Y, Shiozaki H, Tahara H, Inoue M, Oka H, Iihara K, Kadowaki T, Takeichi $M$ and Mori T: Correlation between E-cadherin expression and invasiveness in vitro in a human esophageal cancer cell line. Cancer Res 53: 3421-3426, 1993.

36. Oka H, Shiozaki H, Kobayashi K, Inoue M, Tahara H, Kobayashi T, Takatsuka Y, Matsuyoshi N, Hirano S, Takeichi M, et al: Expression of E-cadherin cell adhesion molecules in human breast cancer tissues and its relationship to metastasis. Cancer Res 53: 1696-1701, 1993.

37. Umbas $R$, Isaacs WB, Bringuier PP, Schaafsma HE, Karthaus HF, Oosterhof GO, Debruyne FM and Schalken JA: Decreased E-cadherin expression is associated with poor prognosis in patients with prostate cancer. Cancer Res 54: 3929-3933, 1994

38. Derksen PW, Liu X, Saridin F, van der Gulden $H$, Zevenhoven J, Evers B, van Beijnum JR, Griffioen AW, Vink J, Krimpenfort $\mathrm{P}$, et al: Somatic inactivation of E-cadherin and p53 in mice leads to metastatic lobular mammary carcinoma through induction of anoikis resistance and angiogenesis. Cancer Cell 10: 437-449, 2006 
39. Frixen UH, Behrens J, Sachs M, Eberle G, Voss B, et al: E-cadherin-mediated cell-cell adhesion prevents invasiveness of human carcinoma cells. J Cell Biol 113: 173-185, 1991.

40. Onder TT, Gupta PB, Mani SA, Yang J, Lander ES and Weinberg RA: Loss of E-cadherin promotes metastasis via multiple downstream transcriptional pathways. Cancer Res 68: 3645-3654, 2008.

41. Baranwal S and Alahari SK: Molecular mechanisms controlling E-cadherin expression in breast cancer. Biochem Biophys Res Commun 384: 6-11, 2009.
42. Yang CC and Wolf DA: Inflamed snail speeds metastasis. Cancer Cell 15: 355-357, 2009.

43. Yang J and Weinberg RA: Epithelial-mesenchymal transition: At the crossroads of development and tumor metastasis. Dev Cell 14: 818-829, 2008.

44. Schmalhofer O, Brabletz S and Brabletz T: E-cadherin, beta-catenin, and ZEB1 in malignant progression of cancer. Cancer Metastasis Rev 28: 151-166, 2009.

45. Nelson WJ and Nusse R: Convergence of Wnt, beta-catenin, and cadherin pathways. Science 303: 1483-1487, 2004. 